\title{
Phénoménologie, anthropologie : Husserl, Heidegger, Sartre
}

\section{Philippe Cabestan}

\section{(2) OpenEdition}

\section{Journals}

Édition électronique

URL : http://journals.openedition.org/alter/390

DOI : 10.4000/alter.390

ISSN : 2558-7927

Éditeur :

Association ALTER, Archives Husserl (CNRS-UMR 8547)

\section{Édition imprimée}

Date de publication : 1 novembre 2015

Pagination : 226-242

ISBN : 978-2-9550449-7-9

ISSN : 1249-8947

\section{Référence électronique}

Philippe Cabestan, «Phénoménologie, anthropologie : Husserl, Heidegger, Sartre », Alter [En ligne], 23 | 2015, mis en ligne le 01 décembre 2017, consulté le 19 avril 2019. URL : http:// journals.openedition.org/alter/390 ; DOI : 10.4000/alter.390 


\title{
PHÉNOMÉNOLOGIE, ANTHROPOLOGIE : HUSSERL, HEIDEGGER, SARTRE
}

\author{
Philippe CEbestEn \\ pour Georges ChErbonneEu
}

Notre propos s'inscrit dans la perspective d'une anthropologie en vue d'une psychiatrie phénoménologique ou DEseinsEnElyse, c'est-àdire d'une compréhension de l'être de l'homme et de la possibilité de son aliénation sous la forme de ce qu'on appelle traditionnellement les maladies mentales et qu'on pourrait également appeler les troubles de l'existence. Ce projet est né de la conviction que la psychiatrie, qu'elle puise ses principes du côté des neurosciences, de la psychanalyse, du béhaviorisme, de la psychologie évolutionnisme, des recherches cognitivo-comportementales, de l'organodynamisme, etc. présuppose, volens nolens, une anthropologie au sens d'une précompréhension de l'existence, qui détermine pour une part non négligeable et sa compréhension des différentes pathologies et leurs traitements. Car il va sans dire qu'on ne soigne pas de la même manière un être humain selon qu'on le tient pour une organisation neuronale, un être pulsionnel ou un existant.

Cependant, un tel projet se heurte à différentes objections plus ou moins décisives. D'une manière très générale, on pourrait tout d'abord rejeter l'idée même d'anthropologie aux prétextes qu'elle charrie des relents essentialistes, qu'elle semble inséparable d'une biologie fixiste pré-darwinienne et qu'elle relève de la métaphysique au mauvais sens du terme ${ }^{1}$. Une autre objection qui vise plus directement la phénoménologie dans son ensemble revient à soutenir que la phénoménologie aussi bien husserlienne que heideggérienne ré-

\footnotetext{
${ }^{1}$ F. Wolff, «La question de l'homme aujourd'hui », Le débEt, no 180, Paris, Gallimard, mai-août 2014, p. 18.
} 
cuse tout projet anthropologique. Jean-Claude Monod parle à ce propos, reprenant une expression de Denis Trierweiler, d'une "phobie de l'anthropologie $»^{2}$. Enfin, on pourrait s'interroger sur la fécondité d'une telle entreprise et sur ses prolongements possibles pour la psychiatrie. Nous voudrions dans les pages qui suivent montrer que, contrairement à ce que soutient Hans Blumenberg dans LE Description de l'homme, ni Husserl ni Heidegger ne formulent un quelconque « interdit anthropologique (Anthropologieverbot)». Et que Sartre pour sa part, même s'il rejette toute idée de nature humaine, fait sienne l'idée d'une anthropologie ontologiquement fondée. Enfin, en prenant pour fil conducteur l'interdit de l'inceste, nous esquisserons de quelle manière il est possible $\mathrm{d}^{\prime}$ articuler in concreto ontologie et anthropologie.

\section{Phénoménologie versus anthropologie dans l'œuvre de Husserl}

C'est notamment dans sa fameuse conférence, intitulée «Phénoménologie et anthropologie $»^{3}$ et prononcée en juin 1931, que Husserl définit la place qui revient d'un point de vue phénoménologique à l'anthropologie. Mais afin de bien saisir l'ambition de ce texte, il convient d'en rappeler les circonstances qui l'ont suscité. On sait que Heidegger offre à la fin de l'année 1926 un exemplaire de Sein und Zeit à Husserl, mais que ce dernier n'entreprend de lire l'ouvrage comme en témoignent ses notes marginales ${ }^{4}$ - que trois années plus tard, donc en 1929. Prenant alors la mesure du fossé qui désormais le sépare de celui qu'il tient pour son disciple, Husserl décide de réagir à ce qu'il interprète comme un triomphe de l'anthropologisme. Il déclare à ce propos : "Alors que la phénoménologie originaire, mûrie en phénoménologie transcendantale, refuse à la science de l'homme, quelle qu'elle soit, toute participation à la fondation de la philosophie et combat à titre d'anthropologisme ou de psychologisme toutes les tentatives qui s'y emploient, il faudrait maintenant que vaille le strict contraire : la philosophie phénoménologique devrait être entièrement

\footnotetext{
${ }^{2}$ J.-C. Monod, " "L'interdit anthropologique" chez Husserl et Heidegger et sa transgression par Blumenberg ", Revue germEnique internEtionEle, $\mathrm{n}^{\circ}$ 10, en ligne, 2009. H. Blumenberg, Description de l'homme, trad. et préface de D. Trierweiler, Paris, Cerf, 2011, p. 14.

${ }^{3}$ E. Husserl, "Phénoménologie et anthropologie ", Notes sur Heidegger, trad. D. Franck, Paris, Minuit, 1993, p. 57-92.

${ }^{4}$ E. Husserl, "Notes marginales de Husserl à Être et temps ", Notes sur Heidegger, trad. N. Depraz, op. cit., p. 9 et $s q$.
} 
reconstruite à partir du DEsein humain $»^{5}$. Il va sans dire que Heidegger est ici explicitement visé même si ces lignes concernent également Whilhelm Dilthey et Max Scheler ${ }^{6}$, sans oublier celui qu'on présente parfois comme son élève, Helmuth Plessner dont Die Stufen des OrgEnischen und der Mensch date de 1928.

Husserl dénonce donc l'anthropologisme de l'analytique existentiale, c'est-à-dire sa prétention à fonder la philosophie en replaçant la conscience dans le champ de l'In-der-Welt-Sein et, du même coup, en subordonnant «la conscience connaissante et réfléchissante à des préalables contingents de la nature humaine et de sa position dans le monde $»^{7}$. Comme le souligne Blumenberg dans sa Description de l'homme, cette prétention de l'anthropologie philosophique n'est jamais qu'un avatar de ce que lui, Husserl, n'a cessé de dénoncer, du moins à partir des Recherches logiques, sous la dénomination de psychologisme, c'est-à-dire la tentation de fonder les mathématiques ou la logique sur la psychologie ${ }^{8}$. À l'anthropologisme, Husserl oppose dans cette conférence le transcendantalisme dont il rapporte la naissance à Descartes et, plus largement, à la lutte des Temps modernes pour une vraie philosophie dont la phénoménologie transcendantale serait la forme actuelle ${ }^{9}$. De ce dernier point de vue, « tout étant mondain et donc aussi mon DEsein en tant qu'homme n'est un existant (DEseiendes) pour moi [ego transcendantal] qu'à titre de contenu $\mathrm{d}^{\prime}$ une certaine aperception empirique ${ }^{10}$. Ainsi $~ «$ la philosophie du DEsein humain ", pour reprendre une formule de Husserl, est non seulement une forme de psychologisme mais également une rechute dans cette naïveté de l'attitude naturelle, que la phénoménologie transcendantale a précisément pour ambition de surmonter. L'anthropologie philosophique aboutit ainsi à cette "géniale absence de scientificité » qui caractérise désormais la pensée de Heidegger pour Husserl ${ }^{11}$.

Une fois admis que l'anthropologie, en tant qu'elle demeure dans l'attitude naturelle, ne saurait se confondre avec la phénoménologie transcendantale, reste toutefois encore à définir rigoureusement la place qui lui revient. Or l'entreprise se révèle particulièrement déli-

\footnotetext{
${ }^{5}$ E. Husserl, « Phénoménologie et anthropologie », Notes sur Heidegger, op. cit., p. 57.

${ }^{6}$ Même si ces deux derniers ne sont plus de ce monde puisque Dilthey meurt en 1911 et Scheler - l'auteur de LEsituEtion de l'homme dEns le monde, ouvrage publié juste après sa mort - en 1928.

${ }^{7}$ H. Blumenberg, Description de l'homme, op. cit., p. 36.

${ }^{8}$ F. Dastur, Husserl. Des mEthémEtiques à l'histoire, Paris, PUF, 1995, p. 26.

${ }^{9}$ E. Husserl «Phénoménologie et anthropologie », Notes sur Heidegger, op. cit., p. 58.

${ }^{10}$ Ibid., p. 67.

${ }^{11}$ E. Husserl, LE Phénoménologie et les fondements des sciences, trad. D. Tiffeneau, Paris, PUF, 1993, p. 176 .
} 
cate dans la mesure où Husserl, dans cette même conférence, reconnaît à l'anthropologie le même statut qu'à la psychologie et, par suite, ce qu'il appelle une "affinité intime » ${ }^{12}$ entre elle et la philosophie transcendantale. En effet, la psychologie n'est pas une science positive parmi d'autres mais elle possède pour Husserl un statut scientifique à part, fondé sur l'abandon nécessaire par le psychologue de sa naïveté naturelle et la découverte d'une intersubjectivité transcendantale. C'est pourquoi, si la phénoménologie ne doit pas se confondre avec la psychologie des psychologues en revanche une nouvelle psychologie ou psychologie phénoménologique pourrait bien in fine se confondre avec une certaine phénoménologie transcendantale. Ce qui est alors en jeu c'est l'idée d'une psychologie transcendantale et, corrélativement, d'une "anthropologie transcendantale». Cette dernière dénomination peut surprendre : de fait, dans un manuscrit inédit, Husserl envisage expressément la possibilité d'une anthropologie transcendantale ${ }^{13}$ - ainsi que $d^{\prime}$ une sociologie transcendantale ${ }^{14}$.

Nous voyons ainsi que l'interdit anthropologique qui pèse sur la phénoménologie husserlienne est tout à fait relatif - ce qu'à vrai dire Hans Blumenberg reconnaît lui-même ${ }^{15}$. Toute la question pour Husserl est de déterminer la place exacte de l'anthropologie et de ne pas y sacrifier l'exigence transcendantale de la phénoménologie ${ }^{16}$. Par ailleurs, si on s'interroge sur la fécondité et les prolongements possibles de cette anthropologie transcendantale dans le champ de la psychiatrie, il suffit de considérer les travaux du dernier Binswanger qui, dans une sorte de retour à Husserl viE Wilhelm Szilasi ${ }^{17}$, aborde la manie à l'aide des concepts de la phénoménologie transcendantale husserlienne. Ainsi Binswanger recourt-il au concept d'apprésentation, tel qu'il est élaboré par la phénoménologie husserliennne de l'intersubjectivité, pour décrire ce qu'il dénomme à propos d'une

\footnotetext{
${ }^{12}$ E. Husserl « Phénoménologie et anthropologie », Notes sur Heidegger, op. cit., p. 73.

${ }^{13}$ E. Husserl, « Anthropologie transcendantale (théologie transcendantale, téléologie) », Manuscrit du sous-groupe E III, Sur l'intersubjectivité, trad. N. Depraz, Paris, PUF, «Epiméthée », 2001, t. II, p. 190.

${ }^{14}$ L. Perreau, Le monde socitl selon Husserl, Phaenomenologica no 209, Dordrecht, Springer, 2013, p. 305.

${ }^{15}$ Une section conclusive de l'ouvrage, comme le rappelle J.-C. Monod, devait montrer comment le Husserl tardif reprend bien le titre d'anthropologie mais ne fait rien de correspondant à une anthropologie, " "L'interdit anthropologique" chez Husserl et Heidegger et sa transgression par Blumenberg », §16.

${ }^{16}$ Tournant le dos à cette exigence, Blumenberg entend de son côté réinscrire l'ego transcendantal dans le monde y compris le monde biologique, J.-C. Monod, " "L'interdit anthropologique" chez Husserl et Heidegger et sa transgression par Blumenberg ", art. cit., §19.

${ }_{17}$ W. Szilasi, Introduction à lE phénoménologie de Husserl, trad. F. Dastur et A. Fournier, Argenteuil, Le Cercle herméneutique, 2011, §40-45.
} 
patiente, Elsa Strauss, et de sa conduite lors d'un épisode de crise maniaque, "une défaillance de l'apprésentation » et une "impossibilité de la constitution d'un monde commun ${ }^{18}$. Nous voyons comment, dans une perspective husserlienne, peuvent s'enchaîner phénoménologie, anthropologie et psychiatrie.

\section{Phénoménologie versus anthropologie dans Sein und Zeit}

Depuis bien des années on s'accorde à considérer que, en assimilant l'analytique du DEsein à une anthropologie philosophique, Husserl méconnaît étrangement l'ambition pourtant explicitement ontologique de Sein und Zeit et commet un contresens que bien d'autres lecteurs renouvèlent par la suite, notamment en France ${ }^{19}$. L'erreur est à vrai dire d'autant plus étrange de la part de Husserl que demeure entre le maître et le disciple un accord manifeste quant aux statuts respectifs de la phénoménologie et de l'anthropologie. En effet, comme nous allons le voir, non seulement il n'est nullement question pour Heidegger de rejeter l'anthropologie mais en outre, à l'instar de Husserl, il envisage son renouvellement à partir de la phénoménologie - de la phénoménologie EiEs l'analytique existentiale, il est vrai.

Afin d'expliciter cet accord, il convient à titre préliminaire de ressaisir les limites, établies par Heidegger lui-même, de l'analytique existentiale et de ne pas confondre d'une part, la problématique de l'entièreté de la structure fondamentale du DEsein et, d'autre part, celle de l'exhEustivité de l'analytique existentiale. En effet, pour Heidegger, la structure du souci - qui rassemble les trois existentiaux de l'existentialité, de la facticité et de la déchéance-dévoile «dans son entièreté (GEnzheit) $»^{20}$ la structure fondEmentEle du DEsein. Aussi cette structure ne saurait-elle être complétée, contrairement à ce que pré-

\footnotetext{
${ }^{18}$ Ainsi, Ludwig Binswanger décrit le comportement d'une patiente, Elsa Strauss, lors de l'une de ses phases maniaques. Cette patiente, au cours d'une promenade, passait devant une église où on célébrait un office religieux. Elle alla vers l'organiste et, pendant qu'il jouait, le félicita pour son jeu et le sollicita pour des leçons d'orgue. Ce qui est ici frappant, c'est l'incapacité de cette malade à se mettre à la place d'autrui, en l'occurrence de l'organiste qu'elle dérange sans s'en rendre compte. L. Binswanger, MEnie et mélEncolie, trad. J.-M. Azorin et Y. Totoyan, Paris, PUF, 1987, p. 80-81.

${ }^{19} \mathrm{~F}$. Dastur note que « le malentendu concernant le véritable statut de l'analyse existentiale dans $\hat{E}$ tre et temps qui a conduit ses premiers lecteurs à y voir la tentative d'une élaboration, d'ailleurs incomplète, d'une anthropologie philosophique, a été grandement facilitée par le fait que la troisième section, proprement "ontologique", de la première partie de cet ouvrage ne fut jamais publiée », F. Dastur, « La critique heideggérienne de l'anthropologisme », Heidegger et lE pensée à venir, Paris, Vrin, 2011, p. 82.

${ }^{20}$ M. Heidegger, Être et temps, trad. F. Vezin, Paris, Gallimard, 1986 (désormais abrégé SZ), §41.
} 
tend par exemple Binswanger dans ses Grundformen und Erkenntnis menschlichen Daseins de $1942^{21}$. Cependant, cette entièreté ne signifie pas que l'être du Dasein et ses différentes déterminations existentiales se réduisent à cette structure première dont, comme on le sait, dérivent bien d'autres déterminations existentiales - telles que la préoccupation (Besorgen), la sollicitude (Fürsorge), l'historialité (Geschichtlichkeit), etc. - qu'il revient à l'analytique existentiale d'établir.

Si on s'interroge, à présent, sur le caractère exhaustif (vollständig) de l'analytique existentiale, il faut immédiatement rappeler que les existentiaux dégagés ne le sont que dans la seule perspective de l'ontologie fondamentale. De ce point de vue, on ne saurait tenir l'analytique existentiale elle-même pour l'exposition exhaustive des déterminations ontologiques du Dasein. C'est du reste ce que reconnaît explicitement Heidegger qui souligne les limites (Grenzen) imposées à l'analytique existentiale par la perspective ontologique qui est la sienne ${ }^{22}$. En d'autres termes, parce que l'analytique du Dasein a pour finalité l'ontologie fondamentale, parce qu'elle est axée «sur l'explication du temps comme horizon transcendantal de la question de l'être ${ }^{23}$, elle se limite à établir les existentiaux relatifs à cette expli-

cation et, comme l'écrit Heidegger, ne saurait « donner une ontologie exhaustive du Dasein ${ }^{24}$.

Il s'ensuit qu'une autre analytique existentiale, c'est-à-dire une analytique existentiale dévoilant à partir de la structure du souci d'autres existentiaux, est possible et, pour ce qui nous concerne, elle se confond avec ce que Heidegger lui-même appelle dans Sein und Zeit « une fondation ontologique de l'anthropologie (eine ontologische Grundlegung der Anthropologie) ${ }^{25}$. C'est pourquoi Heidegger considère que "dans la perspective d'une possible anthropologie, ou tout au moins de sa fondation ontologique », Sein und Zeit offre quelques éléments qui sont loin d'être « inessentiels ${ }^{26}$. Ainsi est envisagée une nouvelle anthropologie qui serait une anthropologie phénoménologique ou encore, écrit Heidegger, « une anthropologie existentiale

\footnotetext{
${ }^{21}$ L. Binswanger, Grundformen und Erkenntnis menschlichen Daseins (1942), Heidelberg, Roland Asanger Verlag, 1993.

${ }^{22}$ L'ontologie fondamentale « $a$, bien sûr, déterminé sans qu'on le dise le cours des considérations faites jusqu'ici, la sélection de phénomènes et les limites imposées à la poursuite de l'analyse », SZ, §42, p. 250.

${ }^{23} \mathrm{SZ}, \S 8$.

${ }^{24} \mathrm{SZ}, \S 5, \mathrm{p} .42$.

${ }^{25} \mathrm{SZ}, \S 42$, p. 250 ,

${ }^{26} \mathrm{SZ}, \S 5, \mathrm{p} .42$.
} 
apriorique $»^{27}$. Cette anthropologie serait radicalement distincte de l'anthropologie d'origine antique et chrétienne dont la définition de l'homme comme animal raisonnable créé à l'image de Dieu est liée à $l^{\prime}$ ontologie de la subsistance ou encore à ce que Heidegger appellera ultérieurement $\mathrm{l}^{\prime}$ ontothéologie ${ }^{28}$. Il n'est donc nullement absurde dans une perspective heideggérienne de se demander ce que pourrait être une anthropologie phénoménologique ou encore une onto-anthropologie. Bref, attribuer à Heidegger l'affirmation d'un quelconque interdit anthropologique est plus que discutable ${ }^{29}$.

$\mathrm{D}^{\prime}$ une manière générale, la phénoménologie heideggérienne ne récuse pas plus l'anthropologie que la sociologie ou la psychologie même si, happée par la question de l'être, elle ne s'est pas développée en direction de ces disciplines. Simplement, selon une démarche analogue à la phénoménologie transcendantale de Husserl, Heidegger affirme la dépendance de ces disciplines vis-à-vis de l'analytique du Dasein en tant qu'elle fixe la structure fondamentale du Dasein et, du même coup, ce à partir de quoi il est possible d'établir les concepts fondamentaux de la psychologie, de la sociologie ou de l'anthropologie. Dans Kant et le problème de la métaphysique, Heidegger reprend même le concept husserlien d'ontologie régionale pour caractériser l'objet de l'anthropologie philosophique et rappelle, à l'instar de Husserl, que cette anthropologie ne peut être considérée comme «le centre de la philosophie ${ }^{30}$. Il convient cependant d'ajouter, afin de ne pas occulter le fossé qui les sépare, que le projet de Heidegger se caractérise par sa volonté de subordonner l'anthropologie à la question de l'être du Dasein en tant qu'ouverture à l'être et, ce faisant, de rompre avec l'anthropologie d'inspiration à la fois grecque et chrétienne dont Husserl aussi bien que Scheler demeurent à ses yeux prisonniers ${ }^{31}$. De ce point de vue, Heidegger refuserait totalement la séparation opérée par Blumenberg - qui est sans doute plus husserlien qu'heideggérien - entre les questionnements anthropologiques et ontologiques, séparation qui repose à vrai dire sur la conviction que la question de l'être «n'existe absolument pas ${ }^{32}$.

\footnotetext{
${ }^{27} \mathrm{SZ}, \S 39$, p. 232.

${ }^{28} \mathrm{SZ}, \S 10$, p. 82.

${ }^{29}$ A. Dirakis, «Penser de l'être, pensée de l'homme », Le débat, no 180, op. cit., p. 99.

${ }^{30}$ J.-C. Monod, " "L'interdit anthropologique" chez Husserl et Heidegger et sa transgression par Blumenberg ", art. cit., §23 ; M. Heidegger, Kant et le problème de la métaphysique, trad. W. Biemel et A. de Waelhens, Paris, Gallimard, 1981, p. 267.

${ }^{31} \mathrm{SZ}, \S 10$, p. 80.

${ }^{32}$ H. Blumenberg, La Description de l'homme, op. cit., p. 208-209.
} 
Phénoménologie, anthropologie : Husserl, Heidegger, Sarte 


\section{Le projet sartrien d'une anthropologie phénoménologique}

Une dépendance analogue de l'anthropologie vis-à-vis de l'ontologie se retrouve mutatis mutandis dans l'œuvre de Sartre, qu'il s'agisse du premier ou du deuxième Sartre, de l'auteur de L'Être et le néant ou de celui de la Critique de la raison dialectique. On sait d'une manière générale l'importance que Sartre accorde à l'anthropologie. Dans une interview accordée en 1966, en réponse à la question : « estce que l'anthropologie épuise tout le champ philosophique? », Sartre soutient «que le champ philosophique c'est l'homme, c'est-à-dire que tout autre problème ne peut être conçu que par rapport à $l^{\prime}$ homme ${ }^{33}$. Par là, Sartre s'oppose nettement à Heidegger : pour l'auteur de Sein und Zeit, une telle affirmation relève de l'oubli de l'être et, corrélativement, de la méconnaissance par l'homme de son propre être, c'est-à-dire de "l'anthropologisme ${ }^{34}$. Cependant, comme nous allons le voir, l'anthropologie - pas plus que l'éthique - ne saurait constituer selon Sartre la philosophie première, et si une anthropologie est possible c'est encore et toujours à partir de l'ontologie en tant qu'il lui revient de poser la question de l'être de l'homme.

En dépit du caractère obvie d'une telle affirmation, il convient de répéter tout d'abord que L'Être et le néant n'est, en toute rigueur, qu'un essai d'ontologie phénoménologique. L'ouvrage ne saurait donc tenir lieu ou envelopper une part d'anthropologie. Sartre luimême le rappelle: " nos recherches présentes ne visent pas à constituer une anthropologie ", lorsqu'il envisage la possibilité d'un poursoi solitaire qui n'aurait jamais fait l'épreuve du regard d'autrui, c'est-à-dire, la possibilité d'un pour-soi qui, comme il l'écrit, "ne serait pas un homme ${ }^{35}$. Que ce point de vue strictement ontologique soit conservé jusqu'au bout de L'Être et le néant, c'est ce dont témoigne entre autres l'explicitation de la responsabilité du pour-soi, qui a suscité autant d'indignation que de contresens. Car la responsabilité y est envisagée dans une perspective strictement ontologique qui laisse délibérément de côté toutes considérations tant juridiques que morales ${ }^{36}$. Mais que la visée de L'Être et le néant ne soit pas anthropolo-

\footnotetext{
${ }^{33}$ Sartre ajoute un peu plus bas : «Le champ philosophique est borné par l'homme » et distingue entre la philosophie pour laquelle l'homme est objet-sujet alors que pour l'anthropologie, les sciences humaines, il est objet. J.-P. Sartre, Situations IX, Paris, Gallimard, 1972, p. 83-84.

${ }^{34} \mathrm{~F}$. Dastur, "La critique heideggérienne de l'anthropologisme », Heidegger et la pensée à venir, op. cit., p. 89.

${ }^{35}$ J.-P. Sartre, L'Être et le néant, Paris Gallimard, 2008 (désormais abrégé EN), p. 322.

${ }^{36} \mathrm{EN}$, p. 598 et $s q$.
} 
gique ne signifie pas que la question anthropologique puisse pour Sartre se poser indépendamment de L'Être et le néant. Au cours de l'introduction de l'Esquisse d'une théorie des émotions (1938), Sartre s'attache à préciser la relation de la psychologie à la phénoménologie et note tout d'abord que la psychologie, y compris dans le cas particulier de la psychologie des émotions, ne peut se passer d'une certaine idée de l'homme, "d'une essence "a priori" d'être humain " - donc d'une anthropologie - qui ne peut être un simple concept empirique, produit de généralisations historiques, et que les sciences positives de la nature seraient bien en peine de lui donner ${ }^{37}$.

Sartre se tourne alors successivement vers Husserl puis vers Heidegger. Du premier, il retient la nécessité de remonter « plus haut que la situation de l'homme dans le monde, jusqu'à la source de l'homme, $\mathrm{du}$ monde et $\mathrm{du}$ psychique: la conscience transcendantale ». Du second, il reprend le projet d'une "herméneutique de l'existence » alias l'analytique existentiale qui «va pouvoir fonder une anthropologie » qui «servira de base à toute psychologie ${ }^{38}$. On dira peutêtre que les deux perspectives sont fondamentalement différentes. Tel n'est pas le cas de Sartre qui les juge au contraire complémentaires, comme le montre à sa manière L'Être et le néant qui conjugue, d'une part, le mouvement analytique de régression ou de remontée à la subjectivité transcendantale, qui aboutit au cogito préréflexif; et, d'autre part, le mouvement synthétique et progressif de dévoilement de l'être de la réalité-humaine en tant que liberté en situation dans le monde, ce que l'Esquisse dénomme l'herméneutique de l'existence ${ }^{39}$. Ainsi, dès 1938, dans une perspective qui se veut à la fois husserlienne et heideggérienne, Sartre envisage l'ontologie phénoménologique comme la condition ou fondement d'une anthropologie et d'une psychologie phénoménologiques.

\section{IV. «Il n'y a pas de nature humaine ${ }^{40}$}

Même si la « découverte » du marxisme modifie certains termes de la problématique, il en va, semble-t-il, de même dans le cadre de la Critique de la raison dialectique. Sartre y reprend en effet sa conception

\footnotetext{
${ }^{37}$ J.-P. Sartre, Esquisse d'une théorie des émotions, Paris, Hermann, 2010, p. 122.

${ }^{38}$ Ibid., p. 14.

${ }^{39}$ La réflexion sur cette double démarche est reprise et approfondie quelques années plus tard dans Questions de méthode. J.-P. Sartre, "La méthode progressive-régressive ", Questions de méthode, Paris, Gallimard, 1960, p. 119-230.

40 J.-P. Sartre, L'existentialisme est un humanisme, Paris, Nagel, 1970, p. 22.
} 
de l'être de l'homme, développée dans L'Être et le néant et popularisée par L'Existentialisme est un humanisme selon laquelle «si Dieu n'existe pas, il y a au moins un être chez qui l'existence précède l'essence, un être qui existe avant de pouvoir être défini par aucun concept et que cet être $c^{\prime}$ est $l^{\prime}$ homme ${ }^{41}$. La Critique de la raison dialectique rejette à son tour l'idée d'une essence ou nature humaine qui déterminerait l'existence des hommes et invoque en outre l'historicité de l'humanité comme la diversité des cultures. Par suite, l'homme ne saurait être l'objet d'un savoir qui définirait une fois pour toutes ce qu'il est de toute éternité ${ }^{2}$. Il faut noter cependant que, mal comprise, cette thèse pourrait aisément être retournée contre le projet d'une anthropologie philosophique. Car en l'absence de toute nature humaine, y compris $\mathrm{d}^{\prime} u$ ne nature humaine définie négativement ${ }^{43}$, $\mathrm{l}^{\prime}$ anthropologie $\mathrm{n}^{\prime} \mathrm{est}$ elle pas condamnée à ces travaux de collectionneurs que dénonce l'Esquisse, c'est-à-dire à multiplier les enquêtes de terrain sans jamais pouvoir s'élever à l'essence ${ }^{44}$ ?

La question est à vrai dire plus difficile qu'on ne le pense parfois et, semble-t-il, met Sartre en contradiction avec lui-même : comment en effet affirmer la précession de l'existence sur l'essence, soutenir qu'il n'y a pas de nature humaine, si l'homme est malgré tout un être qui, par essence, existe librement ${ }^{45}$ ? Afin de prévenir l'objection, il convient de procéder pas à pas et, d'une part, de distinguer entre l'être de l'homme et l'essence de la chose et, d'autre part, de saisir la spécificité du cogito sartrien. Une pierre est un étant intramondain dont le mode d'être est celui de l'en-soi, c'est-à-dire d'un étant qui est ce qu'il est, conformément à son essence que dévoile la liberté en dépassant le monde vers ses possibilités propres. L'essence de cette pierre est alors « le sens de l'objet, la raison de la série d'apparition

\footnotetext{
${ }^{41}$ Ibid., p. 21. Dans L'Être et le néant, Sartre conclut sa discussion de la conception leibnizienne de la liberté par ces mots : «Pour nous, au contraire, Adam ne se définit point par une essence, car l'essence est, pour la réalité-humaine, postérieure à l'existence », op. cit., p. 513. Cf. également, M. Contat et M. Rybalka, "À propos de l'existentialisme : mise au point », Les Écrits de Sartre, Paris, Gallimard, 1970, p. 655.

${ }^{42}$ Par exemple, écrit Sartre, il n'y a pas de «nature humaine » commune aux Muria - un des peuples aborigènes les plus anciens de l'Inde - et à l'homme historique de nos sociétés contemporaines, J.-P. Sartre, Critique de la raison dialectique, Paris, Gallimard, 1985 (désormais abrégé CRD), p. 125.

${ }^{43}$ Notons que Sartre en rejetant l'idée de nature humaine s'inscrit parfaitement dans le prolongement de l'anthropologie philosophique développée au vingtième siècle en Allemagne représentée par Arnold Gehlen (1904-1976) pour qui l'homme est "l'animal non encore fixé », É. Bimbenet, C. Sommer, «Les métaphores de l'humain », Le débat, $\mathrm{n}^{\circ} 180$, op. cit., p. 91.

${ }^{44}$ J.-P. Sartre, Esquisse d'une théorie des émotions, op. cit., p.10.

${ }^{45}$ Heidegger s'en tient à mette le concept d'essence entre guillemets lorsqu'il soutient que " 1 '“essence" du Dasein tient dans son existence », M. Heidegger, SZ, §9.
} 
qui le dévoile ${ }^{46}$. L'être humain est un étant dont le mode d'être est celui du pour-soi, c'est-à-dire d'un existant qui n'est pas ce qu'il est dans la mesure où il a à être et pour qui être c'est avoir étét7. Toutefois, comme le montre L'Être et le néant, il m'est possible à partir $\mathrm{du}$ cogito de dégager sinon l'essence de mon être du moins la structure de mon existence. Sartre s'oppose en effet au cogito cartésien ou husserlien qui est censé livrer une vérité d'essence : "chez l'un [Descartes] nous atteindrons à la liaison de deux natures simples, chez l'autre [Husserl] nous saisirons la structure eidétique de la conscience ». Or, tout ce que je puis demander au cogito sartrien c'est de me découvrir une nécessité de fait. C'est pourquoi Sartre précise que lorsqu'il a décrit la conscience, «il ne pouvait s'agir d'une nature commune à certains individus, mais bien de $m a$ conscience singulière qui, comme ma liberté, est par delà l'essence $»^{48}$. De ce point de vue, la liberté telle que la dévoile $L$ 'Être et le néant, est non pas une essence mais un fait ; elle est "l'étoffe de mon être ${ }^{49}$. Nous comprenons alors que, en toute rigueur, il ne revient pas à L'Être et le néant de dégager l'être de l'homme en général, ce que l'Esquisse appelle "une essence "a priori" d'être humain ", mais de dévoiler l'être de ma conscience en tant que liberté en situation; et c'est à partir de ce premier moment ontophénoménologique, qu'il est possible, en reconnaissant les autres hommes comme autant de libertés en situations, d'élaborer une idée $a$ priori de l'homme, c'est-à-dire une anthropologie.

Si cette articulation de l'ontologie et de l'anthropologie est reprise dans la Critique de la raison dialectique, elle y est toutefois subordonnée, comme nous l'avons dit, à la conviction que le marxisme s'impose désormais "comme la seule anthropologie possible qui doive être à la fois historique et structurelle ${ }^{50}$. Dès lors, la question $\mathrm{du}$ fondement de l'anthropologie devient celle des rapports du marxisme et de l'existentialisme en tant que «fondement humain de $l^{\prime}$ anthropologie marxiste ${ }^{51}$. De ce point de vue, Sartre oppose, d'une part, l'anthropologie positive qui regroupe les différentes sciences de l'homme et qui est un savoir relatif à l'être humain en tant qu'objet et, d'autre part, l'existentialisme en tant qu'anthropologie philosophi-

\footnotetext{
${ }^{46} \mathrm{EN}$, p. 482 et p. 15.

${ }^{47}$ EN, p. 453.

${ }^{48}$ De manière analogue, Sartre dit vouloir, dans la Critique de la raison dialectique, "réintroduire dans le Savoir même et dans l'universalité des concepts, l'indépassable singularité de l'aventure humaine », CRD, p. 129.

${ }^{49}$ EN, p. 483.

${ }^{50}$ CRD, p. 128.

${ }^{51}$ CRD, p. 129.
} 
que. Sans surprise, Sartre établit entre l'une et l'autre un rapport analogue à celui qui lie la phénoménologie husserlienne aux sciences mathématiques de la nature. Et de même que la mécanique classique ne s'interroge pas sur l'espace et le temps qu'elle tient pour des milieux homogènes, de même, souligne Sartre, les sciences de l'homme ne s'interrogent pas sur l'homme ${ }^{52}$. Il revient alors à l'existentialisme de fixer l'être de la réalité humaine et d'en dévoiler les déterminations en procédant non pas sur le modèle de la connaissance des sciences de l'homme, dont les concepts visent des objets, mais par la réflexion compréhensive dont le résultat est l'élucidation des notions les plus élémentaires des sciences de l'homme, telles que le projet, la négation, la transcendance ou encore le besoin « comme être-hors-desoi-dans-le-monde d'un organisme pratique ${ }^{53}$. Sartre qualifie à plusieurs reprises cette connaissance de connaissance "indirecte " 54 , au sens où elle saisit l'homme non pas directement en tant qu'objet mais en faisant le détour par le projet et la praxis individuelle dont la compréhension s'opère dans le cadre de ce qu'il appelle en 1960 l'expérience critique. La thèse demeure inchangée: seul, l'existentialisme est en mesure de donner son véritable fondement à l'anthropologie, c'est-à-dire aux différentes sciences de l'homme.

\section{L'interdit de l'inceste}

Nous avons vu de quelle manière la phénoménologie husserlienne en appelle à un renouvellement de l'anthropologie. Cette anthropologie se distingue alors par son exigence transcendantale au sens phénoménologique du terme, c'est-à-dire par l'exigence de conversion ou, si l'on préfère, de rupture avec l'attitude dite naturelle, qui est au cœur de la réduction phénoménologique. Dans cette même perspective, Heidegger subordonne l'anthropologie ontique à l'analytique du Dasein en tant que dévoilement de la structure fondamentale du Dasein et envisage dans les Zollikoner Seminare la possibilité d'une anthropologie pathologique «articulée selon l'analytique du Dasein ${ }^{55}$. Enfin, nous avons vu que Sartre, tout en rejetant résolument l'idée de nature humaine, ne renonce pas pour autant à donner un fondement humain aux sciences de l'homme, en l'absence

\footnotetext{
${ }^{52}$ CRD, p. 125.

${ }^{53}$ CRD, p. 126.

${ }^{54}$ CRD, p. 126 et p. 132.

${ }^{55}$ M. Heidegger, Séminaires de Zurich, trad. C. Gros, Paris, Gallimard, 2010 (désormais abrégé ZS), p. 189.
} 
duquel ces sciences risquent fort de devenir inhumaines. Cependant, il faut convenir que ces considérations restent bien générales et sans grand rapport avec les recherches et les interrogations actuelles de l'anthropologie. C'est pourquoi nous voudrions envisager à présent la question de l'interdit de l'inceste, question anthropologique s'il en est, afin d'étudier concrètement l'articulation de l'anthropologie et de la phénoménologie.

Il va de soi que Sein und Zeit n'aborde jamais cet aspect de l'existence à la fois individuelle et collective du Dasein, pas plus d'ailleurs qu'il n'y est question de la différence sexuelle. Le Dasein est apparemment asexué et ignore la prohibition de l'inceste. Mais à vrai dire et en toute rigueur, de telles déterminations n'ont pas leur place dans une ontologie fondamentale axée "sur l'explication du temps comme horizon transcendantal de la question de l'être ${ }^{56}$. En revanche, il n'en va plus de même dans une analytique existentiale anthropologiquement - et nous pourrions même ajouter psychiatriquement orientée ${ }^{57}$. Aussi peut-on se demander quelle place l'analytique existentiale est en mesure de reconnaître à un interdit tel que l'interdit de l'inceste : doit-elle considérer cet interdit comme une simple convention, éminemment variable selon les cultures et dont on pourrait même envisager la disparition comme celle de certains interdits relatifs à la pureté et à l'impureté ? Ou bien, au contraire, comme une règle constitutive - et non pas simplement régulatrice - de l'existence humaine, qui structure les relations intersubjectives ainsi que les relations sociales? Manifestement, compte tenu des connaissances actuelles de l'anthropologie - nous pensons aux travaux de Lévi-Strauss repris par Françoise Héritier sur la loi d'exogamie - c'est ce deuxième terme de l'alternative qui s'impose et que l'on peut résumer ainsi : «tous les peuples, malgré quelques exceptions apparentes (en Perse, par exemple, dans la famille royale) prohibent l'union d'une mère et de son fils, d'un père et de sa fille, $d^{\prime}$ un frère et de sa sœur ${ }^{58}$.

Si donc l'interdit de l'inceste n'est pas plus une convention que l'œuvre d'un législateur individuel ou collectif, si donc il ne relève pas d'une explication volontariste ou artificialiste, comment rendre

\footnotetext{
${ }^{56} \mathrm{SZ}, \S 8$.

${ }^{57}$ Nous privilégions ici la perspective heideggérienne mais il serait intéressant d'interroger la place que L'Être et le néant ou la Critique de la raison dialectique seraient (ou ne seraient pas) en mesure d'accorder à l'interdit de l'inceste. Nous avons abordé cette question dans un précédent article, Philippe Cabestan, "L'inconscient est structuré comme un langage. Sartre et le primat lacanien du signifiant ", Les Temps modernes, $n^{\circ}$ 674-675, Sartre avec Freud, Paris, Gallimard, juillet-octobre 2013, p. 34-50.

${ }_{58}$ L. Scubla, Donner la vie, donner la mort. Psychanalyse, anthropologie, philosophie, Lormont, Le Bord de l'eau, 2014, p. 46-47.
} 
compte de l'universelle institution d'un interdit qui fonde le social plus qu'il n'est fondé par lui et qui possède sinon une finalité répondant à une intention consciente du moins un sens? On connaît la solution freudienne, exposée en 1913 dans Totem et tabou. Mais à vrai dire le récit du meurtre du père de la horde originaire n'est guère éclairant dans la mesure où il présuppose ce qu'il est censé fonder. En $\mathrm{d}^{\prime}$ autres termes, pour que des enfants tuent leur père encore faut-il qu'il y ait des enfants et un père, c'est-à-dire un ordre symbolique et non pas simplement une horde dite primitive! La solution de LéviStrauss, qui, non sans raison, subordonne l'interdit de l'inceste à la loi d'exogamie, n'est guère plus satisfaisante. En effet, comme l'écrit Vincent Descombes, elle consiste à «conserver la finalité, mais en la détachant des sujets personnels : c'est la notion d'une "activité inconsciente de l'esprit" » ou encore de structure inconsciente ${ }^{59}$. Or, une telle solution revient - avant l'heure - à naturaliser l'intentionnalité, à naturaliser l'esprit de sorte que les règles de la vie sociale deviennent les régulations inconscientes d'une mystérieuse mécanique cérébrale.

Sans aller plus avant dans l'examen des différentes solutions (nous laissons notamment Lacan de côté60) et sans prétendre apporter une solution définitive, on peut toutefois, du point de vue d'une anthropologie phénoménologique, se demander à quels existentiaux il est possible de rapporter cet interdit. Ce faisant, on s'attache, comme l'écrit Heidegger à propos de l'ethnologie, "à clarifier ontologiquement ce qui a été dévoilé ontiquement ${ }^{61}$, $c^{\prime}$ est-à-dire à ressaisir à partir de l'être de l'étant que nous sommes les déterminations ontiques que nous offre l'anthropologie contemporaine. Ainsi, dans la mesure où, quelle que soit l'ampleur de ses variations culturelles, cet interdit peut être considéré comme une prescription innée, c'est donc à partir de la facticité du Dasein et de son être-jeté qu'il convient alors de ressaisir phénoménologiquement cette règle ou disposition ${ }^{62}$. De fait, le Dasein est un Mitsein, exister signifie coexister. Mais parce

\footnotetext{
${ }^{59}$ V. Descombes, «L'étude anthropologique de l'esprit », La denrée mentale, Paris, Minuit, 1995, p. 83.

${ }^{60}$ Dans L'Éthique de la psychanalyse, Lacan tient l'interdit de l'inceste et le désir de l'inceste pour "la grande trouvaille de Freud" et le meurtre du père primitif pour «le seul mythe dont l'époque moderne ait été capable ", J. Lacan, Séminaire VII, Paris, Seuil, 1986, p. 82 et p. 207-208. ${ }^{61} \mathrm{SZ}, \mathrm{p} .84$.

${ }^{62}$ Innée, cette prescription n'est pas pour autant naturelle, l'idée de nature humaine supposant, comme l'écrit Michel Haar, "l'antithèse métaphysique histoire/nature qui est parfaitement intrahistoriale ». De ce point de vue, l'interdit de l'inceste relèverait alors du pré-historial ou encore du non-historial qui serait à chercher «du côté des limites possibles de l'Histoire à l'intérieur de chacune de ses époques ». M. Haar, Heidegger et l'essence de l'homme, Grenoble, Jérôme Millon, 1990, p. 240.
} 
que cette coexistence n'est pas la simple co-subsistance de monades irrémédiablement séparées les unes des autres, force est d'interroger cette coexistence afin d'en dévoiler les diverses déterminations existentiales. Dans cette perspective, on peut considérer que l'interdiction de l'inceste constitue un existential, au même titre que la sollicitude substitutive ou l'indifférence. Mais contrairement à ces deux dernières déterminations existentiales qui sont de simples possibilités du Dasein dans son rapport inauthentique à autrui, l'interdit de l'inceste est une injonction constitutive du social et régulatrice de la sexualité humaine, à laquelle le Dasein peut bien ne pas se soumettre mais dont la transgression provoque immanquablement de graves troubles. On devine ainsi comment une problématique dite psychopathologique peut se déployer à partir de l'analytique existentiale sans que celle-ci préjuge du contenu de celle-là.

\section{Conclusion}

Dans un ouvrage récent, Albert Piette déclare que «toute référence à Heidegger pour légitimer une anthropologie, suppose de penser contre Heidegger avec Heidegger ${ }^{63}$. Nous avons essayé de montrer que s'il va de soi pour Husserl et pour Heidegger que l'anthropologie ne saurait se substituer à la phénoménologie, cela ne signifie nullement que l'anthropologie soit dépourvue de légitimité scientifique. Bien au contraire, et c'est même à un renouvellement de l'anthropologie que la phénoménologie ouvre la voie. Cette détermination des rapports entre phénoménologie et anthropologie n'est nullement propre à Husserl et Heidegger. Même si la critique sartrienne de l'idée de nature humaine eût pu laisser entendre le contraire, on retrouve bien chez le Sartre de la Critique de la raison dialectique une articulation analogue de l'existentialisme et du marxisme, qui désigne en l'occurrence les sciences de l'homme. Mais remarquons qu'il ne $s^{\prime}$ agit pas alors pour Sartre de reprendre telles quelles les analyse de L'Etre et le néant. Mieux: que le lecteur de L'Être et le néant soit comme désarçonné par la différence de contenu entre L'Être et le néant et la Critique de la raison dialectique ne tient pas tant à une évolution de la pensée de Sartre qui découvre après la seconde guerre mondiale le marxisme qu'à l'exigence d'élaborer une autre ontologie, c'est-à-dire une ontologie en vue du social et de l'historique qui ne peut être

\footnotetext{
${ }^{63}$ A. Piette, Avec Heidegger, contre Heidegger. Introduction à une anthropologie de l'existence, Lausanne, L'Âge d'homme, 2014, p. 51.
} 
identique à une ontologie de l'existence individuelle sans pour autant y être totalement étrangère. De manière analogue, nous avons vu que les déterminations existentiales établies dans Sein und Zeit dépendaient de la perspective dans laquelle elles étaient élaborées et qu'une autre analytique existentiale en vue d'une anthropologie voire d'une anthropo-pathologie était possible.

Toutefois, se demandera-t-on peut-être, pourquoi s'accrocher à l'idée d'une anthropologie phénoménologique et ne pas laisser les anthropologues poursuivre leur tâche en toute indépendance? En d'autres termes, pourquoi maintenir l'exigence transcendantale et prétendre, comme l'écrit Heidegger, «clarifier ontologiquement ce qui a été dévoilé ontiquement ${ }^{64}$ ? Nous pouvons répondre à cette objection à l'aide d'un argument emprunté à Heidegger mais qu'on retrouverait également chez Sartre. En effet, soutenir que l'anthropologie doit procéder empiriquement et tirer ses principes de l'expérience, c'est ignorer qu'il $\mathrm{n}^{\prime} \mathrm{y}$ a pas de recherches empiriques sans présupposés ontologiques et que, comme l'écrit Heidegger, les principes «sont toujours déjà "là" dès lors que du matériel empirique est seulement amassé $»^{65}$. Or, s'il est vrai que l'anthropologie ne peut se passer de principes, il est vraisemblable que ce n'est pas du côté de l'expérience qu'elle doit les rechercher mais bien plutôt du côté d'une analytique existentiale pour autant que celle-ci est en mesure de se poser non pas la question kantienne : qu'est-ce que l'homme? mais la question proprement phénoménologique : quel est le mode d'être de l'homme? En d'autres termes, comprendre l'exigence transcendantale revient à comprendre que «plus originelle que l'homme est la finitude du Dasein en lui ${ }^{66}$.

64 SZ, p. 84.

${ }_{65} \mathrm{SZ}, \mathrm{p} .82$.

${ }^{66}$ M. Heidegger, Kant et le problème de la métaphysique, op.cit., p. 285. 